\title{
Hanging Rotera Modeling by Joining Deformation Result of Space Geometry Objects
}

\author{
Een Ubaningrum, Bagus Juliyanto*, Ahmad Kamsyakawuni, Firdaus Ubaidillah
}

Mathematics Department, Faculty of Mathematics and Sciences, University of Jember, Indonesia

*Corresponding author. Email: bagus.fmipa@unej.ac.id

\begin{abstract}
The hanging rotera is a small lamp covered by a glass lid with a light source from a burning candle or LED (Light Emitting Diode) candle and hung on a support pole that is hooked to the rotera connector. The purpose of this paper are to obtain a models of the various and symmetrical components of the hanging rotera using deformation techniques on space geometric objects. The components of space geometric objects are cylinder, cone, regular hexagon prism, torus, and sphere. This research method determines the size and model the hanging rotera using the deformation technique. The deformation techniques that are cutting, dilatation, interpolation of line segments and curves, and Bezier curves of 2, 3, and 4 degrees. Connecting components of the hanging rotera, we have to notice the radius and the distance of the center of gravity to the corner points of a regular hexagon polygon on each rotera component. The integration of the hanging rotera components as a whole part requires symmetry through the vertical axis which is divided into three parts, namely the rotera part, the connecting part, and the support pole part. We obtain 125 models with five variations on each component of the hanging rotera as the result. The hanging rotera model that we have observed can be visualized using Maple 18.
\end{abstract}

Keywords: Hanging rotera, Deformation technique, Modeling, Space geometric objects.

\section{INTRODUCTION}

The hanging rotera is a small lamp covered by a glass lid with a light source from a burning candle or LED (Light Emitting Diode) candle and hung on a support pole that is hooked to the rotera connector. The rotera component consists of a base, body, and head. The rotera body part has slots that are shaped like a basket, carving, or mirror form. The candle is positioned in the rotera body part.

Some shapes of rotera that exist currently disposed lack of symmetrical, especially in the connection part of the rotera components, whereas the form of supports and connecting the hanging rotera consists of a ring or torus. This case causes the display of the hanging rotera less varied symmetrical so a more varied and symmetrical model of the hanging rotera is needed. The purpose of this paper are to obtain a models the various and symmetrical components of the hanging rotera using deformation techniques on space geometric objects.
The deformation is a process to form the shape of an object, partially or completely [1]. The deformation techniques that are cutting, dilatation, interpolation of line segments and curves, and Bezier curves of 2, 3, and 4 degrees. The research on the use of deformation techniques on space geometric objects has been discussed by $[2,3,4]$.

\section{RESEARCH METHOD}

This research uses the following methods: (a) determining the size of hanging rotera components from the results of deformed space geometry objects such as a cylinder, cone, regular hexagonal prism, torus, sphere, and Bezier curve on 2, 3, and 4 degrees; (b) merging of hanging rotera components via vertical axis that have been divided by three components, such as rotera part, connecting part, and supporting pole part; (c) compiling validation program for hanging rotera modeling using Maple 18. 


\section{RESULT AND ANALYSIS}

\subsection{Data}

The data that were used in this research were regular hexagonal prism, sphere, cylinder, cone, torus, and Bezier curves, for each curve was defined as follows:

a. Regular hexagonal prism with $1 \leq a \leq 9$ units of its base from emphasis to the polygon corner point, and $0,25 \leq t \leq 12$ units of height.

b. Sphere with the center point $P(a, b, c)$ and $0,25 \leq$ $r \leq 3$ units of its radius.

c. The cylinder with $0,1 \leq r \leq 8$ units of its radius and $1 \leq t \leq 25$ units of height.

d. Cone with $1 \leq r \leq 6$ units of its radius and $2 \leq t \leq$ 4 units of height.

e. Torus with the range between torus center point and the center point of the circle was defined as $0,5 \leq$ $R \leq 9$ units and the radius of the rotated circle was defined as $0,25 \leq r \leq 1$ units.

f. Bezier curve with $2 \leq n \leq 4$ degrees.

\subsection{Modeling of Hanging Rotera Parts}

The hanging rotera model consists of three parts, namely rotera, connector, and support. The following are the steps for modeling the hanging rotera.

\subsubsection{Rotera Modeling}

The basic objects used to construct the rotera consist of regular hexagon prism, cylinder, cone, sphere, torus, and Bezier curve. The following are the steps to make the rotera.

a. Rotera base component modeling

The steps to build a rotera base component are as follows:

1. construct the bottom and top base covers using circle planes and regular hexagon polygon planes,

2. construct a cylinder with a central axis parallel to the $z$-axis, with radius $6 \leq r \leq 6,5$ units and height of $0,5 \leq t \leq 1$ units,

3. construct a cone with radius $0<r_{1}<r_{2}$, and $0,5 \leq$ $t \leq 2$ units,

4. construct a regular hexagon prism with the coordinates of the corner points $\left[K_{i}\left(x_{i}, y_{i}, z_{i}\right), K_{i}^{\prime}\left(x_{i}, y_{i}, z_{i}+t\right)\right]$ with $i=1,2, \ldots, 6$. The prism's height is $0,5 \leq t \leq 2$ units, and its center of gravity is $O\left(0,0, z_{1}\right)$ and $O^{\prime}\left(0,0, z_{4}\right)$ respectively. The distance from the center of gravity to the vertices of a regular hexagon polygon is $1 \leq$ $a \leq 7$ units,

5. cut a regular hexagonal prism using a cutting plane $\beta$ parallel to the plane $X O Y$ (Figure 1a),

6. dilate the top cover of a hexagon prism with a scale factor of $k$ and translation as far as $z=z_{1}$ Figure 2b,

7. give variations of curvature on the cylindrical blanket, conical blanket, upright rib of a hexagon prism, and on the sides of a regular hexagon polygon using a $2 \leq n \leq 4$ degree Bezier curves (Figure 1b),

8. roterate the formed Bezier curve about the $z$-axis. If the length of one of the control points on the Bezier curve is $\mathrm{P}_{n}>a$ or $\mathrm{P}_{n}>r$, then it forms an outward curve (convex). If the length of one of the control points on the Bezier curve is $\left(\mathrm{P}_{n}\right)<a$, or $\left(\mathrm{P}_{n}\right)<r$ then it forms an inward curve (concave) (Figure 1b),

9. interpolate the two curves formed using linear interpolation of two line segments or using Bezier shear surface interpolation,

10. duplicate and rotate with an angel of $\theta=60^{\circ}$ on a plane that has been formed, then reflected on the $X O Z$ plane, and the $Y O Z$ plane, so as to form a regular hexagon prism as a result of deformation with sloping sides, convex sides, concave sides, and curved side of Figure 1c,

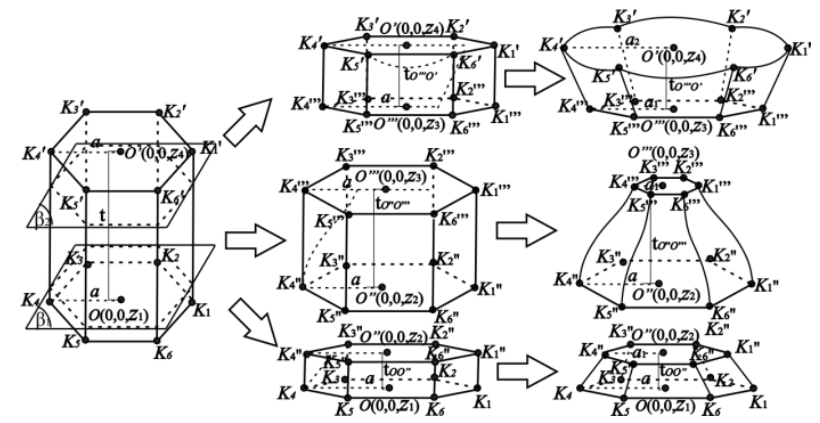

(a) Prism cutting; (b) Prism deformation;

(c) Deformation result

Figure 1 Deformation of a regular hexagon prism.

b. Rotera body component modeling

The rotera body modeling steps are as follows:

1. for example, given a regular hexagon prism with the coordinates of the corner points $\left[K_{i}\left(x_{i}, y_{i}, z_{i}\right), K_{i}^{\prime}\left(x_{i}, y_{i}, z_{i}+t\right)\right.$ with $i=1,2, \ldots, 6$. The prism's height is $1 \leq t \leq 13,5$ units, and its center of gravity is $O\left(0,0, z_{1}\right)$ and $O^{\prime}\left(0,0, z_{4}\right)$ respectively. The distance from the center of gravity 
to the vertices of a regular hexagon polygon is $1 \leq$ $a \leq 6$ units,

2. construct a regular hexagon polygon frame by cutting the prism into three parts. The cut plane used is the secant plane $\beta_{1}$ at the center of gravity $O^{\prime \prime}\left(0,0, z_{2}\right)$ and the section $\beta_{2}$ at the center of gravity $O^{\prime \prime \prime}\left(0,0, z_{3}\right)$ parallel to the $X O Y$ plane with $t_{O O^{\prime \prime}}=t_{O \prime \prime \prime \prime}=0,5$ units (Figure 2a). The coordinates of the corner points of the new regular hexagon polygon prism are $K_{i}{ }^{\prime \prime}\left(x_{i}, y_{i}, z_{2}\right)$ and $K_{i}^{\prime \prime \prime}\left(x_{i}, y_{i}, z_{3}\right)$ with $i=1,2, \ldots, 6$. A regular hexagon prism of $t_{00 \prime \prime}$ is formed as the lower frame, a regular hexagonal prism of $t_{0 \prime \prime \prime \prime \prime}$ as the vertical frame, and a regular hexagonal prism of $t_{0 \prime \prime \prime \prime}$ as the upper frame of Figure 2b,

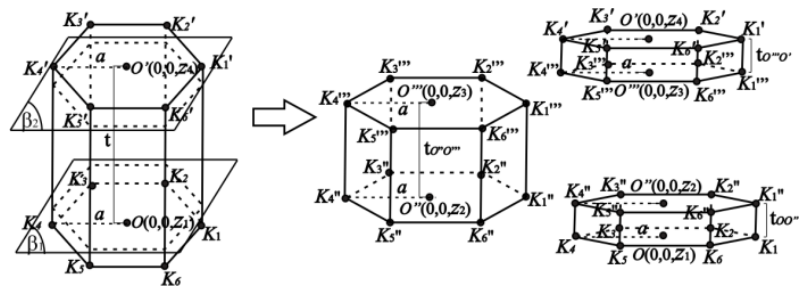

(a) Preliminary data; (b) Cutting a regular hexagon prism

Figure 2 Cutting a regular hexagon prism into three parts

3. construct a vertical frame by cutting a regular hexagon prism $t_{0 \prime \prime \prime \prime \prime}$ using a cutting plane $\alpha$. The cutting edge of a regular hexagon prism is $s=0,5$ units right at the intersection of two side planes with $0 \leq s_{r} \leq \frac{1}{2}$ for the left side, and $\frac{2 a-1}{2} \leq s_{n} \leq a$ for the right side. Duplicate and rotate with $\theta=60^{\circ}$ counterclockwise results from cutting the formed side plane, then reflection on the $X O Z$ plane and the $Y O Z$ plane. Thus, the frame of the rotera bands of Figure 3 is formed,

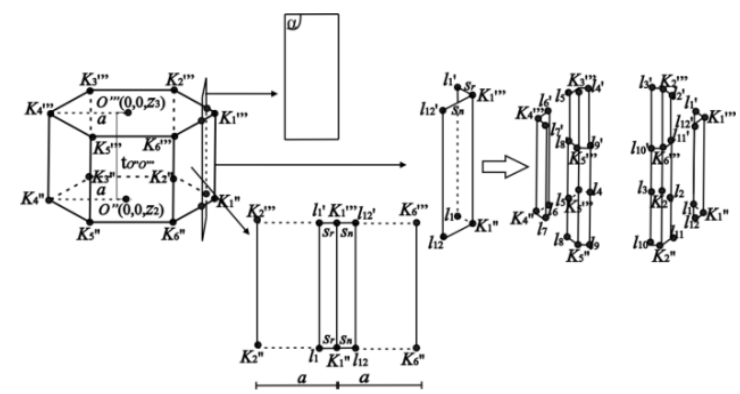

Figure 3 The process of building a stand-up frame.

4. combines the bottom frame, top frame, and upright frame to form a rotera body with an upright frame model,

5. construct the rotera body of the sloping side model as in steps 1 to 4 with a prism height of $1 \leq t \leq 12$ units, and the center of gravity is $O\left(0,0, z_{2}\right)$ and $O^{\prime}\left(0,0, z_{5}\right)$ respectively. The distance from the center of gravity to the vertices of the regular hexagon polygon is $a_{2}$ units for the regular hexagon polygon $K_{i}\left(x_{i}, y_{i}, z_{2}\right)$ and $a_{5}$ units for the regular hexagon polygon $K_{i}{ }^{\prime}\left(x_{i}, y_{i}, z_{5}\right)$, so $0 \leq a_{5} \leq a_{2}$ units and we get the coordinates of the new corner points with different lengths $a$ (Figure 4).

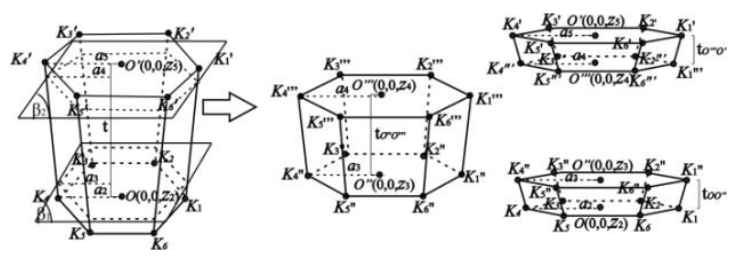

(a) Initial data

(b) Prism cutting

Figure 4 Cutting of a deformed regular hexagon prism.

Determination of the value of $a_{3}$ and $a_{4}$ is done using the principle of similarity of two triangles. Triangle $K_{1}{ }^{\prime} m K_{1}$ where $m$ is the projection of point $K_{1}{ }^{\prime}$ to the extension of point $\overline{O K_{1}}$ line segment, and triangle $K_{1} " n K_{1}$ where $n$ is the projection of point $K_{1}$ " to the extension of point $\overline{O K_{1}}$ line segment (Figure 5). To find the difference between $a_{3}-a_{2}=a_{5}-a_{4}$ is as follows:

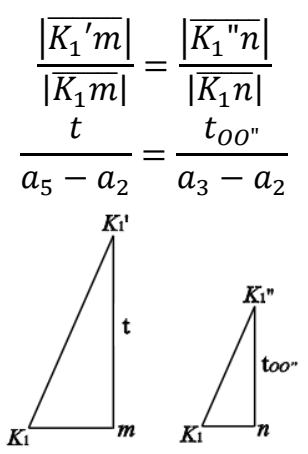

Figure 5 Similarity of triangles.

For example, given the length of $a_{2}=4$ units, $a_{5}=$ 6 units, $z_{2}$ is at coordinates point $O(0,0,1.5), z_{3}$ is at coordinates point $O^{\prime \prime}(0,0,2)$, and $z_{5}$ is at coordinates point $O^{\prime}(0,0,11.5)$ so that $a_{3}-a_{2}=a_{5}-a_{4}=0,1$ units is obtained. Thus, we get $a_{3}=a_{2}+0,1=4+$ $0,1=4,1$ units and $a_{4}=a_{5}-0,1=5,9$ units.

c. Rotera head component modeling

The steps to build the rotera head component are the same as the steps to build the base component, but there are three additions, namely:

1. construct a barrier between the body and the head by interpolating some of the circle plane and the regular hexagon polygons. Suppose the radius of the circle and the distance between the center of gravity and the vertices of the polygons used are $n \leq r, a \leq n+1$ units, 
2. construct a connection between a regular hexagon prism and other shapes by interpolating line segments on a regular hexagon polygon with an inner circle built using a Bezier curve of degrees $n=4$. The initial fixed control point and the final fixed control point are obtained from the vertex $K_{3}$ and the vertex $K_{2}$ on the inner regular hexagon polygon (Figure 6).

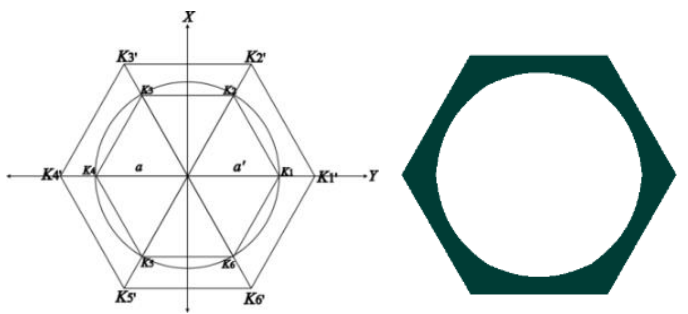

Figure 6 Visualization of the plane of a regular hexagon polygon with partial interpolation of circle shapes on maple 18.

3. construct a torus parallel to the $X O Z$ plane with $0<$ $r<R$

4. construct a cylinder with the central axis parallel to the $\mathrm{z}$-axis with the specified radius and height. Deformation of the cylinder by dividing the cylinder into 16 equal parts vertically. Interpolation on the cylinder section with an odd order of $n=$ $1,3,5, \ldots, 15$ with $\frac{n-1}{16} 2 \pi \leq u \leq \frac{n}{16} 2 \pi$ and $0 \leq v \leq$ 1 (Figure 7)

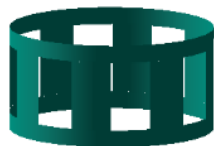

Figure 7 Cylinder blanket cutting visualization.

5. construct a sphere centered on the $x$-axis with a given radius and center. Ball deformation by cutting the ball using a cut the $\alpha$ plane parallel to the $Y O Z$ plane with $0 \leq u \leq 2 \pi$ and $\frac{\pi}{8} \leq v \leq \frac{7}{8} \pi$,

6. contruct a flower cone by dividing the base of the cone into 6 equal parts. Give variations of the curve on the base side of the cut cone to form a curve using a Bezier curve of degree $n=3$. Interpolate the Bezier curve on the side of the base with the vertex of the cone using a Bezier shear curve (Figure 8).
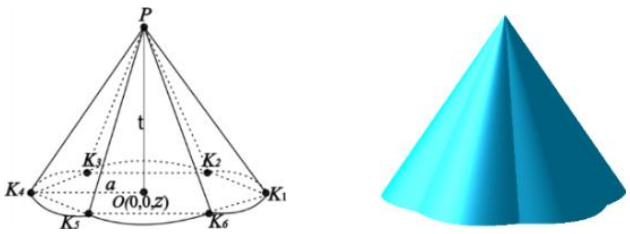

Figure 8 Visualization of The Deformation of a Regular Hexagon Flower Cone on Maple 18
The whole combination of rotera components requires symmetry through the vertical axis with a height of 24,5 $\leq t \leq 25$ units, $t$ as the height of the modeling axis. The combination of the components of the rotera has the order and size of each, namely the height of the rotera base $1 \leq t_{\text {base }} \leq 2,5$ units, the rotera body height $8 \leq t_{\text {body }} \leq 12$ units, and the rotera head height 11,5 $\leq$ $t_{\text {head }} \leq 14,5$ units (Figure 9). The combination of rotary components must pay attention to the radius and distance of the center of gravity to the corner points of a regular hexagon polygon (a) on each rotera component with the following conditions:

1. the radius of the top cover of the rotera base model of the cone and cylinder deformation is greater than the length $a$ of the rotera body ( $r_{\text {base }}>a_{\text {body }}$ ),

2 . the length $a$ on the top cover of the rotera base of variation 1 , and variation 3 is greater than the length of a body ( $a_{\text {base1,3 }}>a_{\text {body }}$ ),

3 . the length $a$ on the top cover of the rotera base of variation 2 and variation 4 is the same as the length of a body $\left(a_{\text {base } 2,4}=a_{\text {body }}\right)$,

4. the length $a$ of the rotera body of the upright side frame model is smaller than the limiting radius $\left(a_{\text {badantegak }}<r_{\text {limiting }}\right)$ and smaller than the limiting length $a$ limiting ( $a_{\text {badantegak }}<a_{\text {limiting }}$ ),

5. the length $a$ of the rotera body of the sloping side frame model is the same as the length $a$ of the lower rotera head $\left(a_{\text {badanmiring }}=a_{\text {kepalabawah }}\right)$.

Based on the above provisions, five types of rota modeling were obtained, namely Type 1 Rotera, Type 2 Rotera, Type 3 Rotera, Type 4 Rotera, and Type 5 Rotera.

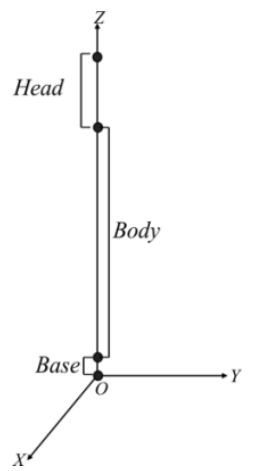

Figure 9 Rotera component modeling axis.

\subsubsection{Support Pole Modeling}

The basic components that make up the support pole consist of a cylinder, a ball, and a torus. The following is a description of the solution to obtain the components of the support pole.

a. construct a torus parallel to the $X O Y$ plane with $0<$ $r<R, r$ is the radius of the circle, $R \mathrm{R}$ is the 
distance between the center of the torus and the center of the circle, $0 \leq u \leq 2 \pi$ and $0 \leq v \leq 2 \pi$ as a support,

b. construct a cylinder with a central axis parallel to the $z$-axis, $r_{\text {tube }}=r_{\text {torus }}$ and a height of $1 \leq t \leq 20$ units as a support pole,

c. contruct a torus parallel to the $Y O Z$ plane with an elliptical path so that there is a difference in the distance between the center of the torus and the center of the circle $R_{1}>R_{2}$. Duplication and reflection of the torus formed on the $X O Z$ plane to form a support pole resulting from the deformation of the torus,

d. construct the base of a regular hexagon polygon shape parallel to the $X O Y$ plane by replacing each side of the polygon using a cylinder with a central axis parallel to the $y$-axis and a cylinder length of $1 \leq y \leq a-1$. Rotation of the cylinder about the $x$ axis with $\theta=60^{\circ}$, followed by reflection on the $X O Z$ plane and the $X O Y$ plane so that a support pole is formed as a result of cylinder deformation. Construct a connection between cylinders using a torus parallel to the $X O Y$ plane for the base of the support and a torus parallel to the $Y O Z$ plane for the support pole with $r_{\text {tabung }}=r_{\text {torus }}($ Figure 10),

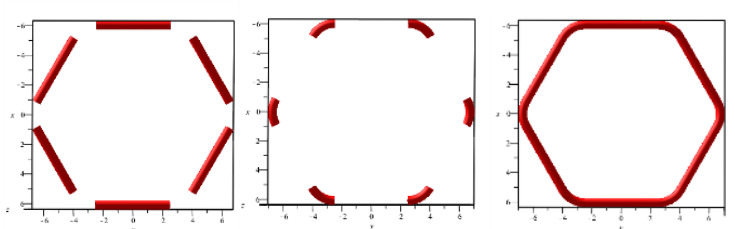

(a) The polygon side of the cylinder; (b) Torus connection; (c) Support base

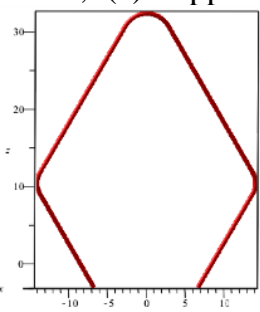

(d) Support pole of deformation cylinder result

Figure 10. Visualization of the base and support post cylinder deformation on Maple 18

e. construct a hemispherical as a cover on the open ends of the torus with $r_{\text {bola }}=r_{\text {torus }}$.

f. combining all components of the supporting poles resulting from the corresponding deformation of the cylinder, torus, and ball to form a variety of support poles.

\subsubsection{Connector Modeling}

The basic components that make up the connector are the cylinder, ball, and torus. The following is an explanation of the solution to get the connecting components.

a. construct a torus parallel to the planes $X O Z, Y O Z$ and $X O Y$ with $0<r<R, r$ is the radius of the circle, $R$ is the distance between the center of the torus and the center of the circle, $0 \leq u \leq 2 \pi, 0 \leq v \leq 2 \pi$ and the specified center,

b. the duplication and reflection of the torus is parallel to the $X O Y$ plane with $\theta=15^{\circ}$ counterclockwise to the $y$-axis, $0 \leq u \leq 2 \pi$, and $\pi \leq v \leq 2 \pi$, the number of the duplication and the reflection are four times each. The translation of each torus is the result of duplication and reflection on the $z$-axis with a height difference of $s=0,62$ units. Thus a connecting body is formed in the form of a Helix curve (Figure 11),

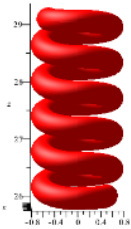

Figure 11 Visualization of the connecting body of the helix curve in maple.

c. construct a cylinder with a central axis parallel to the $z$-axis, $r_{\text {tube }}=r_{\text {torus }}$ and a height of $0,5 \leq t \leq 1$ units as a onnecting hook,

d. construct a cylinder with the central axis parallel to the $y$-axis and and the length of the cylinder is $1 \leq$ $y \leq 2$ units. Rotation of the cylinder about the $x$ axis with $\theta=60^{\circ}$ counterclockwise. Duplication and reflection of the $X O Y$ plane to form a connecting body resulting from cylinder deformation. Build a connection between cylinders using a torus parallel to the YOZ plane with $r_{\text {tube }}=r_{\text {torus }}$,

e. construct a hemispherical as a cover on the open ends of the torus with $r_{\text {bola }}=r_{\text {torus }}$,

f. Combine all connecting components resulting from the corresponding deformation of the cylinder, torus, and sphere to form a variety of connectors.

\subsection{Hanging Rotera Component Combination}

Assembling the hanging rota components as a whole requires symmetry through the vertical axis with a height of $30 \leq t \leq 31$ units, $t$ as the height of the modeling axis. The joining of the hanging rotera parts has their respective order and size, namely the height of the 
rotera $t_{\text {rotera }}=25$ units, the height of connecting $6,5 \leq t_{\text {connector }} \leq 7$ units, and the height of the support pole30 $\leq t_{\text {support pole }} \leq 31$ units. All the results of the modelization of the hanging rota components that have been determined by the model consisting of rota components, support pole components, and connecting components can be combined between components. Thus, 1250 symmetrical and varied models of the hanging rota were obtained Figure 12.

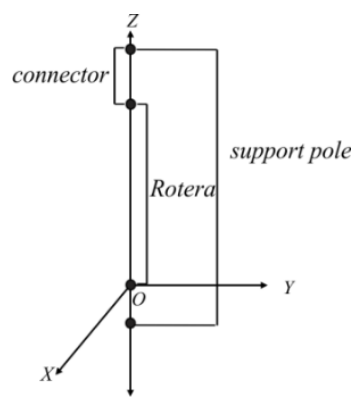

Figur 12 Hanging Rotera Joining Modeling Axis.

\subsection{The Result of The Visualization of The Hanging Rotera}

The results of the visualization of the hanging rotera model on Maple 18 are shown in Table 1.

\section{CONCLUSION}

The result of the research can be summarized as follow:

1. The model of hanging rotera components is construct using deformed results of space geometry objects. The model of hanging rotera components is construct using deformed space geometry objects such as the cylinder, cone, regular hexagonal prism, torus, and sphere. The model of supporting pole components and connecting components are built using space geometry objects such as the cylinder, torus, and sphere. The deformation techniques that are used for hanging rotera model are cutting, dilation, roteration, reflection, roterating the curve, interpolation, and Bezier curve in $n=2,3,4$ degrees. In this research, there are 5 symmetrical and varied variations for each part: rotera part, connecting part, and supporting pole part.
2. Joining of the hanging rotera components is using the symmetry via vertical axis that contains three parts such as the rotera part, connecting part, and supporting pole part. In this research, there are 1250 symmetrical and varied hanging rotera models have been generated.

3. Visualization of hanging rotera modeling result is obtained using Maple 18.

\section{ACKNOWLEDGMENTS}

The author would like to thank an appreciate the official Mathematics' Department of Jember University which provided us this research. And also thank to Bagus Juliyanto, S.Si.,M.Si. as the Main Advisory Lecturer for the support, guidance and funds in carrying out the research.. Furthermore, the authors would like to thank all those who have helped in the implementation of this research.

\section{REFERENCES}

[1] S. Kuang, Geodetic Network Analysis and Optimal Design. Ann Abror Press, Chelsea, Michigan, 2008.

[2] Kusno, Geometri Rancang Bangun Studi Aljabar Vektor Garis, Lingkaran and Ellips, (in Indonesian), Jurusan Matematika Fakultas MIPA, Universitas Jember, 2002.

[3] Kusno, Geometri Rancang Bangun Studi Tentang Desain dan Pemodelan Benda dengan Kurva dan Permukaan Berbantu Komputer, (in Indonesian), Jurusan Matematika Fakultas MIPA, Universitas Jember, 2010 .

[4] N. H. Putri, Kusno, B. Juliyanto, Modelisasi Piala dengan Penggabungan Hasil Deformasi Benda Geometri Ruang, (in Indonesian), Majalah Ilmiah Matematika dan Ilmu Statistika, 2018, 18(1): 23-32. 
Table 1. The Results of hanging rotera modeling

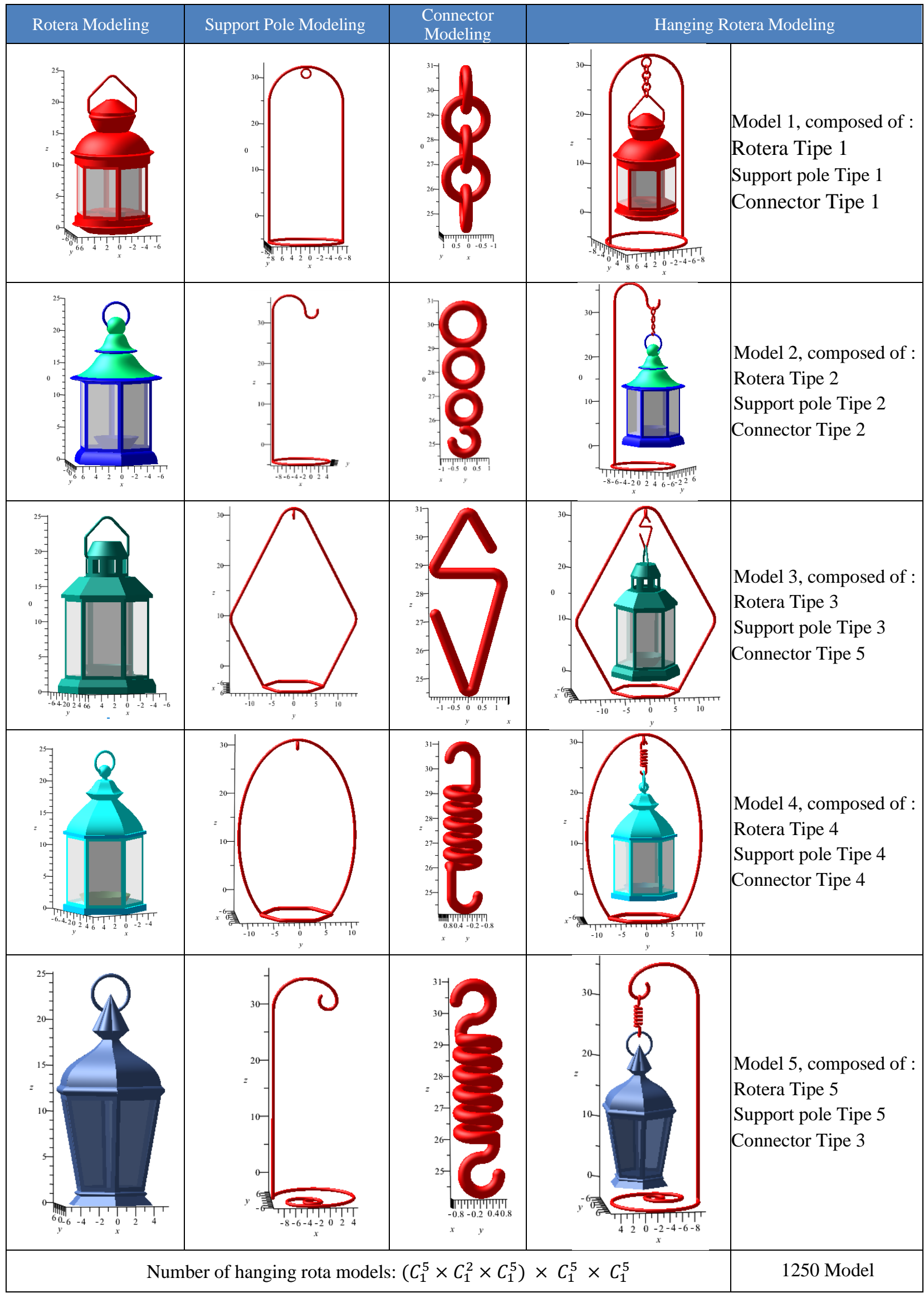

